\title{
A Rare Presentation of Zosteriform Pattern of Cutaneous Metastases from Lung Cancer: A Case Report and Literature Review on Cutaneous Metastases in Non-Small Cell Lung Cancer
}

\section{Matthew Lee ${ }^{*}$, Shikha Sheth1, Syed Ahmad ${ }^{1}$, Qing Chang ${ }^{2}$ and Nagashree Seetharamu ${ }^{3}$}

${ }^{1}$ Division of Internal Medicine, Department of Medicine, North Shore University Hospital-Long Island Jewish Medical Center, 300 Community Dr, Manhasset, NY, USA ${ }^{2}$ Department of Pathology, North Shore University Hospital-Long Island Jewish Medical Center, 300 Community Dr, Manhasset, NY, USA

${ }^{3}$ Department of Oncology, Donald and Barbara Zucker School of Medicine at Hofstra/Northwell, 300 Community Dr, Manhasset, NY, USA

\begin{abstract}
Background: Lung cancer typically metastasizes to the brain, bone, liver and adrenal glands but can rarely metastasize to the skin. It has been reported in only $1-12 \%$ of lung cancer cases and can be an initial finding in newly diagnosed lung cancer patients. These rare findings can be the first indication of a quiescent lung cancer.

Case presentation: This is a case report on a former smoker that initially presented with skin metastases in a zosteriform pattern that was found to be from primary lung adenocarcinoma. He first developed swelling in his left upper extremity and then a rash that was later became vesicular and in ad dermatomal pattern. A subsequent biopsy later revealed this to be from primary adenocarcinoma of the lung.

Conclusion: This case highlights the importance of recognizing skin manifestations in high risk patients with smoking history of lung cancer diagnoses and the need for further research on the mechanism of this etiology and treatment options.
\end{abstract}

Keywords: Lung adenocarcinoma; Cutaneous metastases; Metastasis; Zosteriform

Abbreviations: NSCLC: Non-Small Cell Lung Carcinoma; T: Thoracic; HIV: Human Immunodeficiency Virus; CT: Computed Tomography; TTF-1: Thyroid Transcription Factor-1; CK7: Cytokeratin 7; CK20: Cytokeratin 20; KRAS: Kirsten Rat Sarcoma Viral Oncogene; N/C ratio: Nuclear-Cytoplasmic Ratio; P63: Transformation-Related Protein 63; CDX-2: Caudal-Related Homeobox 2; Gata-3: GATA Binding Protein 3; S100: S100 Proteins; Melan-A: Melanoma Antigen; PSA: Prostate Specific Antigen

\section{Introduction}

Lung cancer is both the second most common malignancy and the leading cause of cancer-associated mortality. It typically metastasizes to the brain, bone, liver and adrenal glands. In rare cases, lung cancer may metastasize to the skin and has been reported in 1-12\% of lung cancer cases [1-4] with one meta analysis reporting 3.4\% of lung cancer patients and $2.8 \%$ in a retrospective study with advanced non-small cell lung cancer (NSCLC) as the initial presentation. Overall, in men who present with skin metastases, the most common primary site is melanoma (32.3\%) and lung cancer (24\%) [4]. Furthermore, 25\% of these cases presented initially with cutaneous findings before the diagnosis of primary lung cancer was made [5]. Clinically, lung cancer could only be initially recognized as cutaneous metastases while the primary lung cancer remains quiescent. Here we present a rare initial presentation of skin metastases in zosteriform pattern from a primary lung adenocarcinoma and the importance of recognizing its significance.

\section{Case Presentation}

A 78-year-old male with a history of atrial fibrillation, type 2 diabetes and former significant tobacco use of 94 pack years, presented with a one month history of left sided neck pain. The pain was progressive and he subsequently developed left upper extremity swelling along with a nonpruritic and non painful rash involving the neck, left anterior chest and back that started expanding. In the hospital, the patient reported no recent infections or travels, fevers, dyspnea, or weight changes. On physical exam, patient was noted to have a firm, indurated mass on his left upper back along with a vesicular rash in a T3-4 dermatomal pattern concerning for shingles. Laboratory findings were significant for leukocytosis, normal calcium level and HIV was negative. Ultrasound confirmed a mural thrombus in the left internal jugular vein along with left cervical, supraclavicular and axillary lymphadenopathy, and he was started on Lovenox for anticoagulation. CT Chest revealed a right upper lobe heterogeneous enhancing mass $(7.2 \times 4.9 \times 5.9 \mathrm{~cm})$ concerning for malignancy. A left axillary node biopsy was performed which revealed

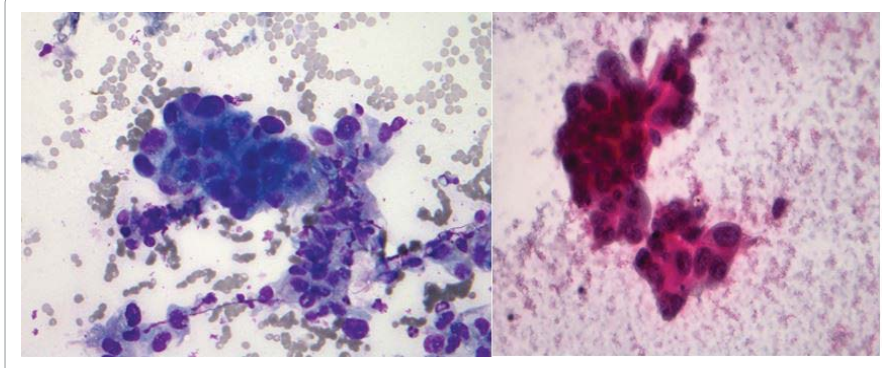

Figure 1: The left axillary lymph node showed metastatic poorly differentiated carcinoma. The smears are composed of crowded 3-dimensional groups and single lying pleomorphic malignant cells with enlarged, irregular nuclei containing prominent nucleoli and vacuolated cytoplasm in a background of lymphocytes.

*Corresponding author: Matthew Lee, Resident, Division of Internal Medicine Department of Medicine, North Shore University Hospital-Long Island Jewish Medical Center, 300 Community Dr, Manhasset, NY, 11030, USA, Tel: +1 516-6276622; E-mail: mlee37@northwell.edu

Received January 04, 2019; Accepted January 25, 2019; Published January 29, 2019

Citation: Lee M, Sheth S, Ahmad S, Chang Q, Seetharamu N (2019) A Rare Presentation of Zosteriform Pattern of Cutaneous Metastases from Lung Cancer A Case Report and Literature Review on Cutaneous Metastases in Non-Small Cell Lung Cancer. J Cancer Sci Ther 11: 025-027. doi: 10.4172/1948-5956.1000578

Copyright: $\odot 2019$ Lee M, et al. This is an open-access article distributed under the terms of the Creative Commons Attribution License, which permits unrestricted use, distribution, and reproduction in any medium, provided the original author and source are credited. 

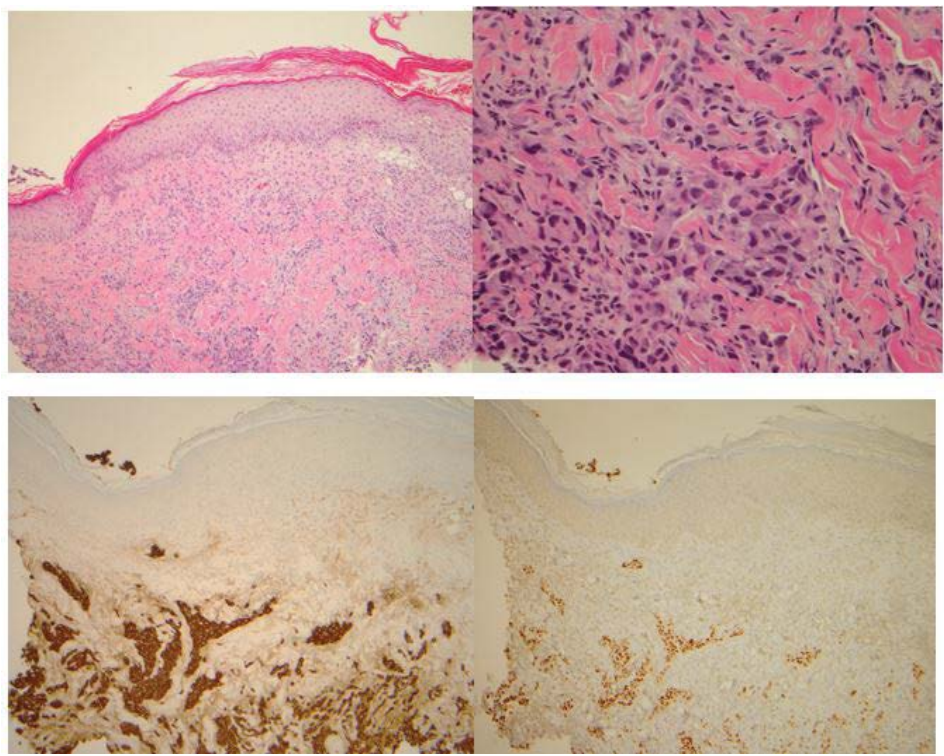

Figure 2: The epidermis consists of epidermis and dermis only. The dermis is infiltrated by poorly differentiated carcinoma in a solid nest and trabecular pattern. The tumor cells have a hyperchromatic and angulated nucleus with a high N/C ratio and marked pleomorphism. Neoplastic cells are positive for CK7 and TTF-1, weakly positive for P63 and CK20, negative for CDX-2, Gata-3, S100, Melan-A and PSA. The histopathological and immunocytochemical findings in conjunction with clinical history of lung mass are those of metastatic poorly differentiated adenocarcinoma from lung origin.

metastatic poorly differentiated carcinoma (Figure 1) and a subsequent skin biopsy showed primary adenocarcinoma of the lung (Figure 2). Immunohistochemical staining was positive for TTF-1, a marker that is sensitive and specific for primary lung adenocarcinoma, as well for CK7, CK20 and next generation sequence with KRAS mutation which supports a metastatic adenocarcinoma from the lungs. ALK and EGFR mutations were not present and PDL-1 levels were low at $1 \%$. The patient was discharged with outpatient medical oncology follow-up but opted for in-patient hospice and passed away 3 months later.

\section{Discussion}

Overall, cutaneous metastases have been reported in only 0.7$9 \%$ of patients with cancer [1] but can be a silent indicator of visceral malignancies occurring between 50 and 70 years with a median age of 46.5-years-old [6]. The primary sites of carcinomas with cutaneous metastases in men are melanoma, lungs, colon, oral cavity and kidney and gastric but in a series of studies, it has been reported that skin is the 13th most common site of lung cancer metastasis [7]. Cutaneous metastasis can present in a variety of forms ranging from nodules, ulcerations or bullae with nodules being the most common seen as high as $82.5 \%$ in one study [8]. They can grow and necrotize and ulcerate along with colors from red to blue-black and appear in groups [2-4].

Although cancers such as liver cancer have random distribution for cutaneous metastasis, in terms of lung cancer, there is predilection for the chest and supradiaphragmatic area. Moreover, cancers of the upper lobes of the lungs have more tendency to metastasize to the skin [9]. As for our patient, he presented with cutaneous metastases on his chest. Furthermore, in our case, the patient presented with zosteriform skin metastasis which is very rare. It has been reported in a meta-analysis of 56 cases of zosteriform skin metastasis that only five of the cases were lung cancer with diagnosis to zosteriform skin metastasis varying from at time of diagnosis to 1 year [10]. The mechanism however is still unknown but some theories that have been reported have been either related to lymphatic systemic with focal cancer embolism leading to retrograde flow and skin metastases $[11,12]$ to surgical implantation
[13] to defective immunological response with prior herpes zoster infection [14]. However, the exact mechanism for the tropism for skin in lung cancer is still unknown and further studies are needed to understand this phenomenon.

Generally, though cutaneous metastatic lesions from their physical appearance are indistinguishable from their origin of carcinomas. Histology is important in order to distinguish these metastasis. TTF-1 immunostain is characteristic of primary lung cancer and mutations including EGFR, ALK and KRAS mutations in adenocarcinomas specifically. In this patient, his biopsy was positive for TTF-1 and KRAS mutation confirming the patient's primary malignancy as lung adenocarcinoma. There is limited reports on the association of histological type of lung cancer and occurence of cutaneous metastasis. There has been a prior report of a KRAS-mutated lung adenocarcinoma with cutaneous metastasis but not with a zosteriform presentation [4]. Other reports have shown conflicting results where some have demonstrated large cell lung carcinoma with a greater tendency to metastasize to the skin and lung adenocarcinoma the least and others showing adenocarcinoma having the greatest tendency and large cell the least $[15,16]$. More research is needed on examining this relationship further and whether there is prognostic implications. It has been shown in prior studies that poor differentiation and upper lobe carcinomas increases the risk of cutaneous metastasis possibly related to the anatomical hematogenous and lymphatic flow $[3,16]$.

It has been shown that the presence of cutaneous metastasis indicates a poorer prognosis. It typically signifies an aggressive primary lung carcinoma and and an average survival seen with three to five months as such as in our patient compared to 10 months for patients who did not have cutaneous metastases $[1,8]$. Another factor that can be contributing to a worse prognosis is that the average time interval between diagnosis of primary malignancy and the cutaneous metastases can range from 18 to 33 months and only $50 \%$ surviving within the first 6 months of the first appearance of the cutaneous lesions $[1,8]$. 
Citation: Lee M, Sheth S, Ahmad S, Chang Q, Seetharamu N (2019) A Rare Presentation of Zosteriform Pattern of Cutaneous Metastases from Lung Cancer: A Case Report and Literature Review on Cutaneous Metastases in Non-Small Cell Lung Cancer. J Cancer Sci Ther 11: 025-027. doi: 10.4172/1948-5956.1000578

There is no established guidelines for cutaneous metastases in NSCLC and specifically lung adenocarcinoma but it generally includes surgery alone or combined with palliative chemotherapy and with radiation therapy if there is severe pain or bleeding [4]. One study by Ambrogi et al., showed that presented two cases treated with surgery and chemotherapy where one was alive at 74 months and other at 8 months since time of diagnosis [17]. In a larger case series, 6 patients had surgery and of those 4 also had chemotherapy and 2 with concomitant radiation therapy. The average survival of 12.5 months after diagnosis and suggested that surgery can increase survival [18]. If multiple cutaneous metastases are present, chemotherapy is the main management and response can be monitored from the lesion sizes [19]. Agents used as shown by Kamble et al., include cisplatin, adriamycin, vincristine, cyclophosphamide regimens but survival rates are still poor at only 6.5 to 8 months after chemotherapy possibly from low perfusion of chemotherapies to the skin $[13,20,21]$. Another case report similar to our case in a patient also with zosteriform skin metastasis in a lung cancer patient had treated the patient with targeted therapy with second line erlotinib with no response and then salvage chemotherapy with pemetrexed for third line therapy but still had disease progression [10]. Lastly there is also reports on the use of tyrosine kinase inhibitors (TKIs) such as gefitinib or erlotinib in EGFR mutated patients [22,23] and ALK gene rearrangement with crizotinib.

\section{Conclusion}

This rare case of metastatic lung adenocarcinoma presenting initially with skin metastases in a zosteriform dermatome pattern highlights the importance of recognizing new skin findings in high risk patients such as those with significant smoking history as one of the first clinical manifestation of a possible occult malignancy.

\section{Declarations}

\section{Acknowledgement}

The authors would like to thank all members of the study team, the patient's family, and Northwell Health System and North Shore University Hospital for their support.

\section{Funding}

No funding or grants to support this research.

\section{Availability of data}

The datasets supporting the conclusions of this article are included within the article.

\section{Authors' contributions}

SS and SA provided the patient information and case, ML and NS prepared the manuscript and analysis. QC provided the pathology analysis and pictures. All authors read and approved the final manuscript.

\section{Ethics approval and consent to participate}

In accordance with the Declaration of Helsinki, informed written and verbal consent to participate and share publication of this case report and any potentiallyidentifying information/images was obtained by the patient's legal representative his wife. As per the Ethics and IRB committee at the Office of the Human Research Protection Program at Feinstein Institute for Medical Research at Northwell Health able to proceed with publishing the case report and exempt according to their Human Research Protection Program Guidance Document for case reports less than 3 subjects.

\section{Competing interests}

The authors have no potential conflicts of interest interest exist with any companies/organizations whose products or services may be discussed in this article.

\section{References}

1. Hussein MR (2010) Skin metastasis: A pathologist's perspective. J Cutan Pathol 37: e1-20.

2. Hussain J, Westerveld D, Sattari M (2016) Cutaneous metastasis: A rare herald of a silent cancer. BMJ Case Rep p: bcr2015213734.

3. Mollet TW, Garcia CA, Koester G (2009) Skin metastases from lung cancer Dermatol Online J 15: 1.

4. Liao H, Wu S, Karbowitz SR, Morgenstern N, Rose DR (2014) Cutaneous metastasis as an initial presentation of lung adenocarcinoma with KRAS mutation: A case report and literature review. Stem Cell Investig 1: 6.

5. Savoia P, Fava P, Deboli T, Quaglino P, Bernengo MG (2009) Zosteriform cutaneous metastases: A literature meta-analysis and a clinical report of three melanoma cases. Dermatol Surg 35: 1355-1363.

6. Sarid D, Wigler N, Gutkin Z, Merimsky O, Leider-Trejo L, et al. (2004) Cutaneous and subcutaneous metastases of rectal cancer. Int J Clin Oncol 9: 202-205.

7. Kamisawa T, Takahashi M, Nakajima H, Egawa N (2006) Gastrointestinal: Zosteriform metastases to the skin. J Gastroenterol Hepatol 21: 620.

8. Yogi V, Singh DP, Ahirwar M, Mandloi V (2018) Spectrum of cutaneous metastasis from internal malignancies a study of clinico-pathological and survival evaluation of 126 patients. Int J Sci Res 7: 2277-8179.

9. Kondras K, Zalewska A, Janowski P, Kordek R (2006) Cutaneous multifocal melanoma metastases clinically resembling herpes zoster. J Eur Acad Dermatol Venereol 20: 470-472.

10. Li WH, Tu CY, Hsieh TC, Wu PY (2012) Zosteriform skin metastasis of lung cancer. Chest 142: 1652-1654.

11. Zalaudek I, Leinweber B, Richtig E, Smolle J, Hofmann-Wellenhof R (2003) Cutaneous zosteriform melanoma metastases arising after herpes zoster infection: A case report and review of the literature. Melanoma Res 13: 635-639.

12. Schoenlaub P, Sarraux A, Grosshans E, Heid E, Cribier B (2001) Survival after cutaneous metastasis: A study of 200 cases. Ann Dermatol Venereol 128: 1310-1315.

13. Hidaka T, Ishii Y, Kitamura S (1996) Clinical features of skin metastasis from lung cancer. Intern Med 35: 459-462.

14. Molina-Garrido MJ, Guillén-Ponce C, Soto-Martínez JL, Martínez Y, Sevila C, et al. (2006) Cutaneous metastases of lung cancer. Clin Transl Oncol 8: 330-333.

15. Krathen RA, Orengo IF, Rosen T (2003) Cutaneous metastasis: A metaanalysis of data. South Med J 96: 164-167.

16. Song Z, Lin B, Shao L, Zhang Y (2012) Cutaneous metastasis as an initial presentation in advanced non-small cell lung cancer and its poor survival prognosis. J Cancer Res Clin Oncol 138: 1613-1617.

17. Ambrogi V, Tonini G, Mineo TC (2001) Prolonged survival after extracrania metastasectomy from synchronous resectable lung cancer. Ann Surg Oncol 8: 663-666.

18. Dreizen S, Dhingra H, Chiuten D, Umsawasdi T, Valdivieso M (1986) Cutaneous and subcutaneous metastases of lung cancer. Postgrad Med 80: 111-116.

19. Kamble R, Kumar L, Kochupillai V, Sharma A, Sandoo MS, et al. (1995) Cutaneous metastases of lung cancer. Postgrad Med J 71: 741-743.

20. Terashima T, Kanaqawa M (1994) Lung cancer with skin metastasis. Chest 106: $1448-1450$.

21. Douillard JY, Shepherd FA, Hirsh V, Mok T, Socinski MA, et al. (2010) Molecular predictors of outcome with gefitinib and docetaxel in previously treated nonsmall-cell lung cancer: Data from the randomized phase III interest trial. J Clin Oncol 28: 744-752.

22. Lynch TJ, Bell DW, Sordella R, Gurubhagavatula S, Okimoto RA, et al. (2004) Activating mutations in the epidermal growth factor receptor underlying responsiveness of non-small-cell lung cancer to gefitinib. N Engl J Med 350 2129-2139.

23. Kwak EL, Bang YJ, Camidge DR, Shaw AT, Solomon B, et al. (2010) Anaplastic lymphoma kinase inhibition in non-small-cell lung cancer. N Eng J Med 363: 1693-1703. 